\title{
Interference-Aware Spectrum Sharing Techniques for Next Generation Wireless Networks
}

\author{
Authors Marwa Khalid Qaraqe, Ziad Bouida, Mohamed Abdallah, Mohamed-Slim \\ Alouini \\ Institutions Texas A\&M University at Qatar, Doha, Qatar \\ King Abdullah University of Science and Technology, Jeddah, \\ Saudi Arabia \\ e mail marwa@tamu.edu
}

Background: Reliable high-speed data communication that supports multimedia application for both indoor and outdoor mobile users is a fundamental requirement for next generation wireless networks and requires a dense deployment of physically coexisting network architectures. Due to the limited spectrum availability, a novel interference-aware spectrum-sharing concept is introduced where networks that suffer from congested spectrums (secondarynetworks) are allowed to share the spectrum with other networks with available spectrum (primary-networks) under the condition that limited interference occurs to primary networks.

Objective: Multiple-antenna and adaptive rate can be utilized as a powerefficient technique for improving the data rate of the secondary link while satisfying the interference constraint of the primary link by allowing the secondary user to adapt its transmitting antenna, power, and rate according to the channel state information.

Methods: Two adaptive schemes are proposed using multiple-antenna transmit diversity and adaptive modulation in order to increase the spectral-efficiency of the secondary link while maintaining minimum interference with the primary. Both the switching efficient scheme (SES) and bandwidth efficient scheme (BES) use the scan-and-wait combining antenna technique (SWC) where there is a secondary transmission only when a branch with an acceptable performance is found; else the data is buffered.

Results: In both these schemes the constellation size and selected transmit branch are determined to minimized the average number of switches and achieve the highest spectral efficiency given a minimum bit-error-rate (BER), fading conditions, and peak interference constraint. For delayed sensitive applications, two schemes using power control are used: SES-PC and BES-PC. In these schemes the secondary transmitter sends data using a nominal power level, which is optimized to minimize the average delay. Several numerical examples show that the BES scheme increases the capacity of the secondary link.

Conclusion: The SES and BES schemes reach high spectral efficiency and BER performance at the expense of an increased delay. The SES-PC and BES$P C$ minimize the average delay, satisfy the BER, and maintain a high spectral efficiency. The proposed power optimization and power control processes minimize the delay and the dropping probability especially if we extend the presented work to a multiuser scenario. 